\title{
Interactions between GABAergic and Cholinergic Processes in the Nucleus Pontis Oralis: Neuronal Mechanisms Controlling Active (Rapid Eye Movement) Sleep and Wakefulness
}

\author{
Ming-Chu Xi, ${ }^{1,2}$ Francisco R. Morales, ${ }^{1,3}$ and Michael H. Chase ${ }^{1,2}$ \\ ${ }^{1}$ WebSciences International, Los Angeles, California 90024, ${ }^{2}$ Department of Physiology and the Brain Research Institute, School of Medicine, University of \\ California, Los Angeles, California 90095, and ${ }^{3}$ Departamento de Fisiologia, Facultad de Medicina, Universidad de la Republica, Montevideo 11800, \\ Uruguay
}

The cholinergic system within the nucleus pontis oralis (NPO) of the pontine tegmentum is critically involved in the generation of active (rapid eye movement) sleep. Previously, we demonstrated that a GABAergic system in the NPO also plays an important role in the control of the behavioral states of wakefulness as well as active sleep. The present study examined interactions between these two neuronal systems vis-à-vis the occurrence of these behavioral states. Accordingly, cholinergic and GABAergic agonists and antagonists were injected into the NPO, and their combined effects on sleep and waking states of chronic, unanesthetized cats were examined. Microin-

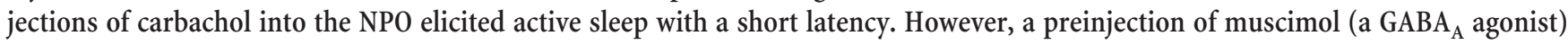
completely blocked the active sleep-inducing effects of carbachol. The induction of active sleep by carbachol was also suppressed by a subsequent injection of muscimol. On the other hand, the microinjection of scopolamine (a muscarinic receptor antagonist) did not block the induction of active sleep by bicuculline ( $\mathrm{GABA}_{\mathrm{A}}$ antagonist). We conclude that the excitatory cholinergic control of NPO neurons that are involved in the generation of active sleep is gated by a pontine GABAergic system that exerts its effects postsynaptically by inhibiting NPO neurons, resulting in the suppression of active sleep and the generation of wakefulness. In the absence of the activation of this GABAergic gating mechanism, active sleep occurs. These results reveal that specific interactions between cholinergic and GABAergic processes in the NPO play a critical role in the generation of active sleep and wakefulness.

Key words: REM sleep; wakefulness; GABA; acetylcholine; pontine reticular formation; cat

\section{Introduction}

Pontine cholinergic mechanisms play a critical role in the generation of active [rapid eye movement (REM)] sleep (for review, see Chase and Morales 1990; Steriade and McCarley, 1990; Jones, 1991; Baghdoyan, 1997; Rye 1997; Siegel, 2000). Since the initial demonstration by George et al. (1964) that brainstem cholinergic mechanisms are involved in the induction of active sleep, numerous studies have confirmed that cholinergic agonists, when injected into the nucleus pontis oralis (NPO), which is located in the pontine reticular formation, are capable of inducing an active sleep-like state with all of its attendant physiological patterns of activity (Baghdoyan et al., 1984, 1989, 1993; Vanni-Mercier et al., 1989; Yamamoto et al., 1990; Yamuy et al., 1993).

Supporting the role of cholinergic mechanisms in the genera-

Received Feb. 27, 2004; revised Sept. 2, 2004; accepted 0ct. 13, 2004.

This work was supported by United States Public Health Service Grants NS 23426, NS 09999, MH 43362, HL 60296, and AG 04307. We thank Dr. J. K. Engelhardt for his critical comments regarding this manuscript and Oscar Ramos, Andrui Nazarian, Kristian Werneid, and Kim-tan Nguyen for their technical assistance.

Correspondence should be addressed to Dr. Michael H. Chase, WebSciences International, 1251 Westwood Boulevard, Los Angeles, CA 90024. E-mail: mchase@websciences.org.

DOI:10.1523/JNEUROSCI.1987-04.2004

Copyright $(\odot 2004$ Society for Neuroscience $\quad 0270-6474 / 04 / 2410670-09 \$ 15.00 / 0$ tion of active sleep are findings of an increase in acetylcholine (ACh) release in this region during spontaneous active sleep (Kodama et al., 1990), as well as during carbachol-induced active sleep (Lydic et al., 1991). In addition, the induction of active sleep by cholinergic agonists within the NPO is blocked by muscarinic receptor antagonists (Baghdoyan et al., 1989; VelazquezMoctezuma et al., 1991). To date, active sleep induced by cholinergic agonists provides the most useful pharmacological model of active sleep (Steriade and McCarley, 1990).

We have recently provided data demonstrating that GABAergic inhibitory processes in the NPO also play an important role in the control of the behavioral states of active sleep and wakefulness (Xi et al., 1999a,b, 2001). These data emanated from studies in chronic cats in which prolonged periods of wakefulness and increased motor activity were induced after the injection of GABA, $\mathrm{GABA}_{\mathrm{A}}$, and $\mathrm{GABA}_{\mathrm{B}}$ agonists into the NPO. The injection of the corresponding antagonists into the NPO resulted in the rapid induction of long episodes of active sleep (Xi et al., 1999a,b, 2001). In addition, we found that the administration, into the $\mathrm{NPO}$, of antisense against glutamic acid decarboxylase (GAD), which interferes with the synthesis of GABA and therefore reduces endogenous GABA content in this area, produced ex- 
tended periods of decreased wakefulness and increased active sleep (Xi et al., 1999b).

We therefore hypothesize that the generation and maintenance of active sleep as well as wakefulness depend on the interaction between cholinergic and GABAergic systems in the NPO: specifically, that the excitatory cholinergic activation of NPO neurons is gated by a pontine GABAergic inhibitory system. To test this hypothesis, we examined the behavioral responses of chronic, unanesthetized cats after the injection, into the NPO, of cholinergic and GABAergic receptor agonists and antagonists separately as well as in selected combinations. The results of these experiments indicate that interactions between cholinergic and GABAergic neurotransmitters exist in the NPO and that they play a critical role in the generation of active sleep and wakefulness.

\section{Materials and Methods}

Seven adult cats, obtained from and determined to be in good health by the University of California Los Angeles (UCLA) Division of Laboratory Animal Medicine, were used in the present study. All experiments were conducted in accordance with the Guide for the Care and Use of Laboratory Animals and approved by the Animal Research Committee of the UCLA Office for the Protection of Research Subjects.

Animals and surgical procedures. The animals were prepared for monitoring the behavioral states of sleep and wakefulness and for drug administration, as described previously (Yamuy et al., 1993). Briefly, under isofluorane anesthesia, using sterile surgical procedures, the cats were implanted with electrodes for recording the electroencephalogram, the electro-oculogram, and the electromyogram. A Winchester plug, connected to these electrodes, and a chronic head-restraining device were bonded to the calvarium with acrylic cement. A hole $\sim 5 \mathrm{~mm}$ in diameter, which was drilled in the calvarium overlying the cerebellar cortex, was covered with bone wax. During experimentation, this hole provided for the access for a cannula that was used for drug microinjection. After surgery, antibiotics were administrated both systemically and topically.

After recovery from surgery, all cats were adapted to the recording environment by placing them in a head-restraining apparatus each day for 2 weeks. Thereafter, whenever they were placed in the headrestraining apparatus, the animals exhibited spontaneous periods of wakefulness (W), quiet [nonrapid eye movement (NREM)] sleep (QS), and active sleep (AS).

Drug administration. The effective NPO site in each cat was defined as the stereotaxic coordinates (Berman, 1968) within the rostral pontine reticular formation wherein an injection of carbachol $(0.25 \mu \mathrm{l}, 22 \mathrm{~mm}$ in saline) induced active sleep with a latency shorter than $4 \mathrm{~min}$. Once a short latency site was determined, the stereotaxic coordinates for that site were used for all injections during subsequent experiments in that cat. The combined unilateral injections of cholinergic and GABAergic agonists and antagonists into the NPO were performed during experimental sessions, which were conducted between 10:00 A.M. and 4:00 P.M.

In the first set of experiments, muscimol, a $\mathrm{GABA}_{\mathrm{A}}$ receptor agonist $(0.25 \mu \mathrm{l}, 100 \mu \mathrm{M}$ to $10 \mathrm{~mm}$ in saline), was injected first; this injection was then followed by an injection of carbachol $(0.25 \mu \mathrm{l}, 22 \mathrm{~mm}$ in saline) or a control injection of saline $(0.25 \mu \mathrm{l})$, followed by an injection of carbachol. In the second set of experiments, carbachol was injected first, and it was followed by an injection of muscimol ( $10 \mathrm{~mm}$ ) or carbachol, followed by saline. In the third set of experiments, scopolamine, a muscarine receptor antagonist $(0.25 \mu \mathrm{l}, 10 \mathrm{~mm}$ in saline), was injected first; it was followed by an injection of bicuculline, a $\mathrm{GABA}_{\mathrm{A}}$ receptor antagonist $(0.25 \mu \mathrm{l}, 10 \mathrm{~mm}$ in saline), or scopolamine, followed by an injection of carbachol. The doses of the preceding drugs were based on those reported previously to be most effective in the NPO of the cat (Baghdoyan et al., 1989; Xi et al., 1999b, 2001, Yamuy et al., 1993). In all experiments, dual injections were performed using a $2 \mu \mathrm{l}$ Hamilton syringe with an interval of 20-30 min between injections. The injection syringe was connected to a remote-controlled hydraulic micropositioner so that the animals were not disturbed by the injection procedure. Drugs were delivered over a period of $1 \mathrm{~min}$. The order of all three sets of experiments was counter- balanced so as to control for possible order effects. In addition, there was a minimum of $4 \mathrm{~d}$ between experimental sessions in each cat. A maximum of six sets of microinjections were made into the same site on either the left or the right side of each animal. No abnormal behavior was observed; in addition, control patterns of sleep and waking were recorded after the injection sessions in each cat to ensure that the effects of injections were reversible and that the injections did not damage the NPO.

Recording and data analysis. Polygraphic records were digitized and recorded using a Power Macintosh computer (Apple Computer, Cupertino, CA) running SuperScope II software (GW Instruments, Somerville, MA). The behavioral states of W, QS, and AS were scored according to standard polygraphic and behavioral criteria (Ursin and Sterman, 1981). Polygraphic recordings, which were divided into $30 \mathrm{sec}$ epochs, were used to construct hypnograms. The following dependent variables were determined based on polygraphic data and behavioral observations during each recording session: (1) percentage of time spent in wakefulness, quiet sleep, and active sleep during the first hour after the last injection, as well as throughout the $3 \mathrm{hr}$ recording period; (2) latency to the onset of the first episode of each behavioral state, as measured from the time of the beginning of the last injection; (3) number of episodes of each behavioral state per hour (frequency); and (4) duration of the longest episode of each behavioral state. Experimental data are expressed as means \pm SEM. The statistical significance of the difference between sample means was evaluated using the two-tailed unpaired Student's $t$ test and an ANOVA. The criterion chosen to discard the null hypothesis was $p<0.05$.

Histological location of injection sites. At the conclusion of the microinjection experiments, the site of drug injection was marked with $0.5 \mu \mathrm{l}$ of a $2 \%$ solution of Chicago sky blue dye in $0.5 \mathrm{M} \mathrm{Na}$-acetate. The animal was then killed with an overdose of Nembutal and perfused with saline, followed by a solution of $10 \%$ formaldehyde. Serial sections of brainstem

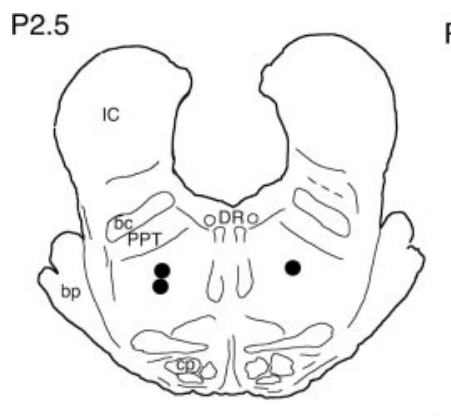

P3.0

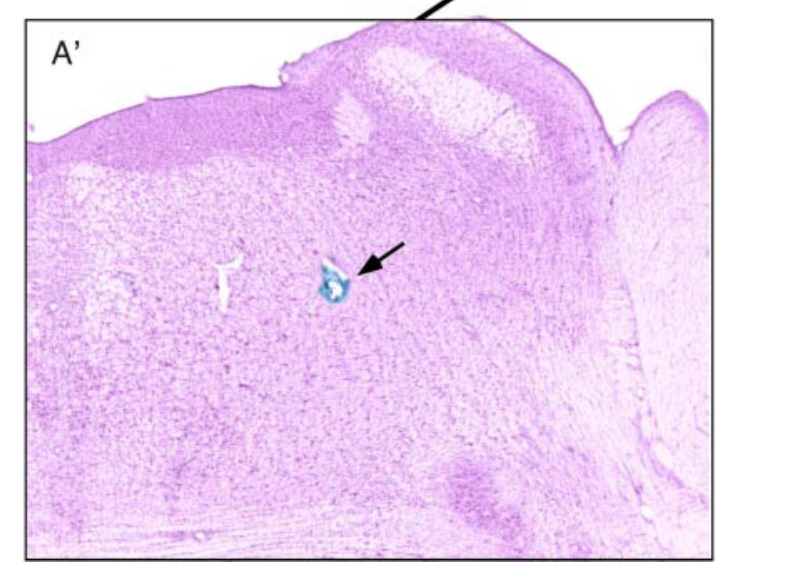

Figure 1. Anatomical location of injection sites $(n=8)$ in the nucleus pontis oralis. The effective injections of both cholinergic and GABAergic agonists and antagonists were all within the NPO. Two schematic frontal planes of the cat brainstem are illustrated at levels posterior (P) 2.5 and $P$ 3.0, respectively. Circles indicate sites where injections were delivered to the left and right side, respectively. The boxed area $(A)$ is enlarged and presented in a photomicrograph $\left(A^{\prime}\right)$. The arrow points to the injection site, which is marked with the Chicago sky blue dye. bc, Brachium conjunctivum; bp, brachium pontis; $c p$, cerebral peduncle; DR, dorsal raphe nucleus; FTP, paralemniscal tegmental field; IC, inferior colliculus. 
tissue were examined to verify the injection sites. Histological studies revealed that the effective injection sites were all located within the NPO and that there was no evidence of damage to the surrounding tissue (Fig. 1).

\section{Results}

A combination of cholinergic and GABAergic agonists and antagonists were microinjected, unilaterally, into the NPO, and their effects on the behavioral states of sleep and wakefulness were examined. As described in Material and Methods, three sets of experiments of combined injections of cholinergic and GABAergic agonists and antagonists were performed: (1) muscimol followed by carbachol, (2) carbachol followed by muscimol, and (3) scopolamine followed by bicuculline. In addition, saline (as a control) was injected into the same site that received injections of the preceding drugs. The following sections describe, quantitatively, the effects of the combined injections of these drugs on the states of wakefulness, quiet sleep, and active sleep.

\section{Effects of injection of muscimol followed by carbachol}

Figure 2 presents representative polygraphic recordings and hypnograms from experiment sessions in a cat that show the effects on sleep and wakefulness of the combined injection of muscimol and carbachol. As reported previously, the microinjection of muscimol into the NPO during quiet sleep induced, with a short
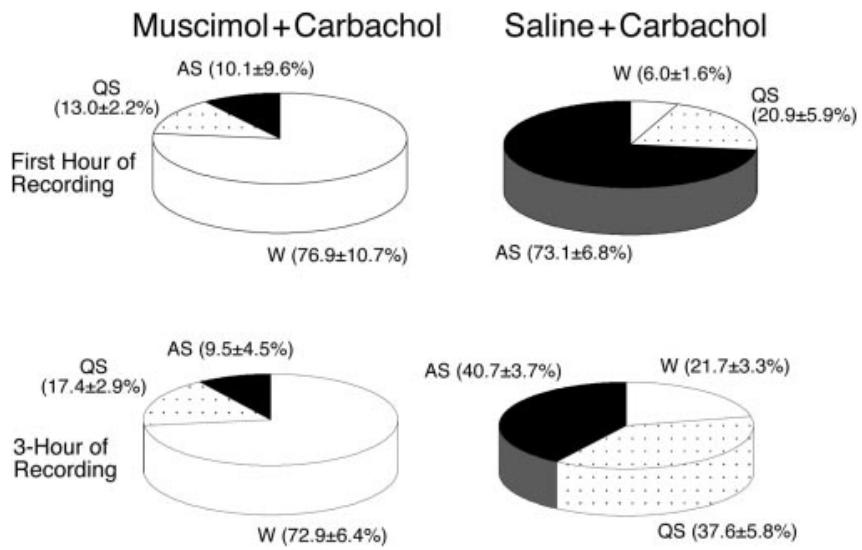

Figure 3. Pie charts presenting the mean percentage of time spent in wakefulness, quiet sleep, and active sleep during the first hour (top) and the $3 \mathrm{hr}$ recording period (bottom) after the combined injection of muscimol and carbachol or saline and carbachol, respectively. Data within parentheses are means \pm SEM. Compared with the percentages observed after the injection of saline and carbachol $(n=6)$, the injection of muscimol and carbachol significantly increased the percentage of time spent in wakefulness $(p<0.01)$ and significantly decreased the percentage of time spent in active sleep $(p<0.01)$ during the first hour of recording. During the entire $3 \mathrm{hr}$ recording period, the injection of muscimol and carbachol also induced significant changes in the percentage of time spent in wakefulness $(p<0.01)$ and active sleep $(p<0.01)$.

\section{A. Microinjection of Muscimol and Carbachol}

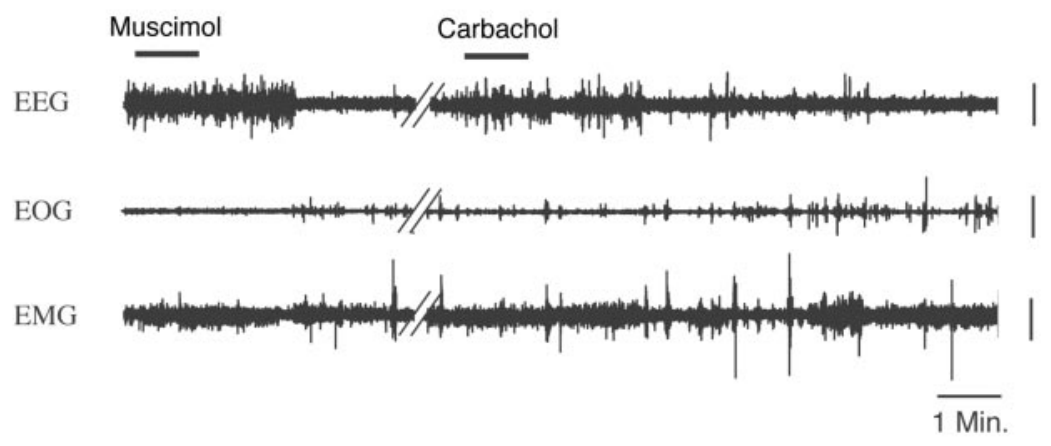

B. Muscimol and Carbachol

C. Saline and Carbachol
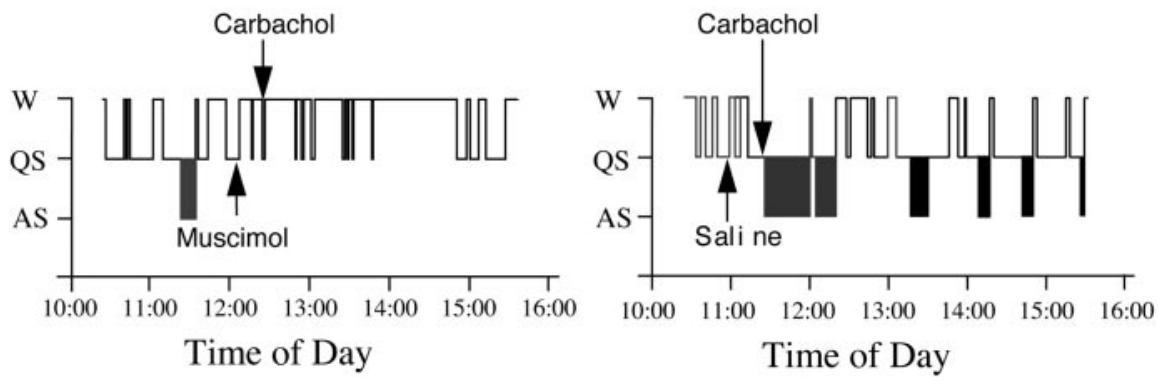

Figure 2. An injection of muscimol into the NPO blocked carbachol-induced active sleep. $A$, Representative polygraphic recordings after the combined injection of muscimol and carbachol. Note that the injection of muscimol induced wakefulness with atency of $2.3 \mathrm{~min}$. The injection of muscimol was performed during a spontaneous episode of quiet sleep. Twenty-one minutes

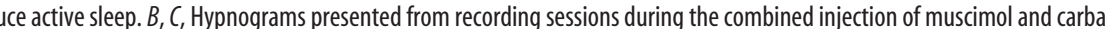
episode of active sleep (C). However, when muscimol was injected into the NPO, 21 min before the injection of carbachol, it blocked the induction of active sleep by carbachol $(B)$. Control injections of saline into the same area of the NPO did not block active action of this drug and was not an artifact of the injection procedure (C). EEG, Electroencephalogram; EOG, electro-oculogram; EMG, electromyogram. Vertical calibration bars, $100 \mu \mathrm{V}$. latency, wakefulness; the microinjection of carbachol into the NPO alone, on the other hand, induced a long-lasting episode of active sleep (Baghdoyan et al., 1984, 1993; Xi et al., 1999a,b, 2001). However, when muscimol was injected into the NPO, 21 min before the injection of carbachol, it blocked the active sleepinducing effects of carbachol (Fig. $2 A, B$ ). The control injection of saline into the same area of the NPO did not block the induction of active sleep by a subsequent injection of carbachol, indicating that the effect of muscimol was produced by the pharmacological action of this drug and was not an artifact of the injection procedure (Fig. 2C).

The effects of the combined injection of muscimol (10 mM) and carbachol $(22 \mathrm{~mm})$ on the percentage of time that the cats spent in sleep and waking states during the first hour after injection of carbachol are presented in Figure 3. Compared with the percentage observed after the injection of saline and carbachol $(n=6)$, the injection of muscimol and carbachol reduced the time spent in active sleep by $86.2 \%(n=7 ; p<0.01)$ and significantly increased the time spent in wakefulness by $1181.7 \%(p<$ 0.01 ). To determine the effect of the injection of muscimol for an extended period, the percentages of time spent in sleep and waking states was examined for a $3 \mathrm{hr}$ recording session after the injection of carbachol (Fig. 3). During the $3 \mathrm{hr}$ recording period, changes in the percentage of time spent in wakefulness, quiet, and active sleep were similar to those observed during the first hour, 


\section{A. Latency of Behavioral States}

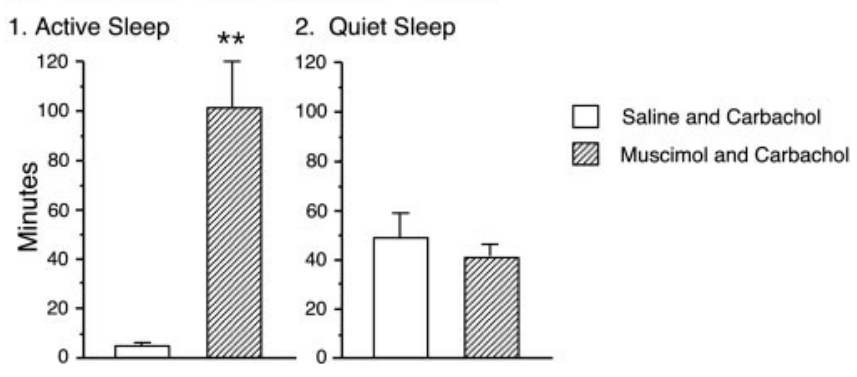

\section{B. Frequency of Behavioral States}

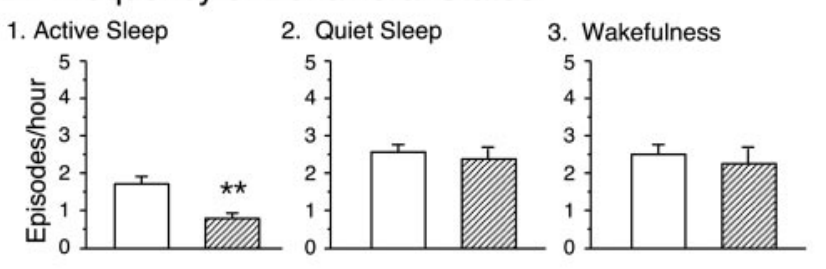

\section{Duration of Behavioral States}

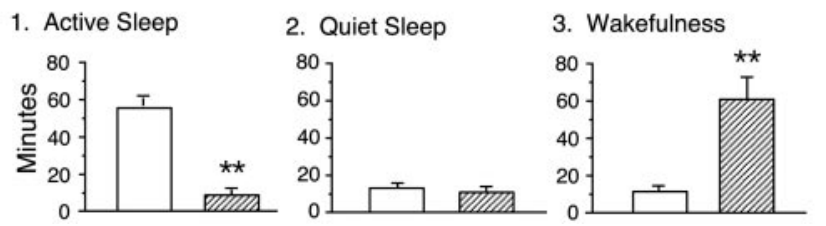

Figure 4. Effects of the combined injection of muscimol and carbachol on the latency, frequency, and duration of the longest episodes of active sleep, quiet sleep, and wakefulness. Each bar represents the mean value; error bars indicate the SEM of each population. $A$, The effect of the injection of muscimol and carbachol on the mean latency of active sleep. The latency was measured from the second injection to the onset of the first episode of the indicated behavioral state. Injections of muscimol and carbachol significantly increased the mean latency of active sleep. $B$, The effect of the injection of muscimol and carbachol on the mean frequency (the number of episodes of a specific behavioral state per hour) of active sleep (B1), quiet sleep (B2), and wakefulness $(B 3)$. The injection of muscimol and carbachol significantly reduced the frequency of active sleep. $C$, The effect of the injection of muscimol and carbachol on the duration of the longest episode of active sleep (C1), quiet sleep (C2), and wakefulness (C3). The injection of muscimol and carbachol significantly decreased the mean duration of active sleep and increased the mean duration of wakefulness. Asterisks indicate the levels of statistical significance of the difference between means; ${ }^{* *} p<0.01$.

which indicates a long duration for the effects provided by muscimol.

The effects of the combined microinjection of muscimol and carbachol were also assessed by measuring the latency to the onset of the first episode of active sleep after the injection of carbachol (Fig. 4A). The mean latency of active sleep was $102.0 \pm 22.9 \mathrm{~min}$ $(n=7)$, which was significantly longer compared with that of active sleep that occurred in conjunction with saline-injected controls (saline plus carbachol, $3.6 \pm 0.8 \mathrm{~min} ; n=6$ ). Because most injections of carbachol were made during wakefulness, which was induced by a preceding injection of muscimol, there was no measure of the latency of wakefulness.

The effects of muscimol, when followed by an injection of carbachol into the NPO, on the frequency of sleep and waking states during the $3 \mathrm{hr}$ recording period are shown in Figure $4 \mathrm{~B}$. It should be noted that, because of the long duration of wakefulness induced by the injection of muscimol and the facilitation of active sleep that followed by the injection of carbachol, the frequency of active sleep was significantly different between muscimol plus carbachol and saline plus carbachol sessions [active sleep, $0.7 \pm$
A. Muscimol and Carbachol

B. Muscimol and Carbachol
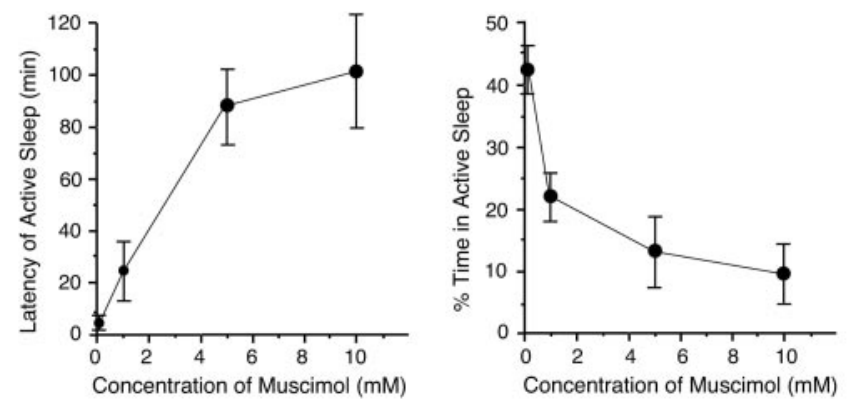

Figure 5. Dose-dependent effects of a preinjection of muscimol on the mean latency to the first episode of active sleep $(A)$ and the mean percentage of time spent in active sleep $(B)$ during the $3 \mathrm{hr}$ recording period after an injection of carbachol $(22 \mathrm{~mm})$. All four doses of muscimol were injected in a saline solution of $0.25 \mu$ l. Data were obtained from 18 injections ( $100 \mu \mathrm{m}, n=3$; $1 \mathrm{~mm}, n=4 ; 5 \mathrm{~mm}, n=4 ; 10 \mathrm{~mm}, n=7)$. Each point presents the mean for each dose of muscimol; error bars indicate the SEM.

0.2 episodes/hr (muscimol plus carbachol) vs $1.7 \pm 0.2$ episodes/hr (saline plus carbachol); $p<0.01$ ]. The frequency (episodes per hour) of wakefulness and quiet sleep after the injection of muscimol and carbachol was not significantly different from that after the injection of saline and carbachol.

The effect on the duration of the longest episodes of each behavioral state was examined after the injection of muscimol and carbachol (Fig. 4C). Muscimol significantly increased the duration of wakefulness (muscimol plus carbachol, $61.8 \pm 10.6$ min vs saline plus carbachol, $11.2 \pm 2.6 \mathrm{~min} ; p<0.01$ ). These data are consistent with the above findings that demonstrated that muscimol increased the time spent in wakefulness. The mean duration of active sleep was significantly reduced after the injection of muscimol and carbachol compared with that after the injection of saline and carbachol (muscimol plus carbachol, $8.6 \pm 3.7 \mathrm{~min}$ vs saline plus carbachol, $55.7 \pm 7.7 \mathrm{~min} ; p<0.01$ ).

Muscimol was also injected into the NPO at four different concentrations, ranging from $100 \mu \mathrm{M}$ to $10 \mathrm{mM}$, which was followed by the injection of carbachol ( $22 \mathrm{~mm})$. As shown in Figure $5 \mathrm{~A}$, the preinjection of muscimol produced a dose-dependent increase in the latency to the onset of the first episode of active sleep after the injection of carbachol. The injection of the lowest concentration of muscimol $(100 \mu \mathrm{M})$ did not produce a significant effect on the latency to active sleep after the injection of carbachol; the latency to active sleep was comparable with that after the injection of saline and carbachol (muscimol plus carbachol, $3.9 \pm 1.1 \mathrm{~min}$ vs saline plus carbachol, $3.6 \pm 0.8 \mathrm{~min}$ ). The latency to active sleep increased steadily with increasing concentrations of muscimol. A positive correlation was found between the mean latency of active sleep after the injection of carbachol and the concentration of muscimol $(100 \mu \mathrm{M}, 3.9 \pm 1.1 \mathrm{~min}, n=$ $3 ; 1 \mathrm{~mm}, 23.3 \pm 12.1 \mathrm{~min}, n=4 ; 5 \mathrm{~mm}, 89.3 \pm 14.0 \mathrm{~min}, n=4 ; 10$ $\mathrm{mM}, 102.0 \pm 22.9 \mathrm{~min}, n=7 ; r=0.84 ; p<0.01$; Spearman rank correlation coefficient)

Increasing the concentration of a preinjection of muscimol also produced a dose-dependent decrease in the percentage of time spent in active sleep for the $3 \mathrm{hr}$ recording session after the injection of carbachol (Fig. 5B). Injections of the lowest dose of muscimol $(100 \mu \mathrm{M})$ did not produce a significant decrease in the percentage of time spent in active sleep (muscimol plus carbachol, $43.3 \pm 4.1 \%, n=3$ vs saline plus carbachol, $40.7 \pm 3.7 \%$, $n=6 ; p>0.05)$. However, the mean percentage of time spent in active sleep was significantly reduced after the injection of a $1 \mathrm{~mm}$ 
A. Microinjection of Carbachol and Muscimol

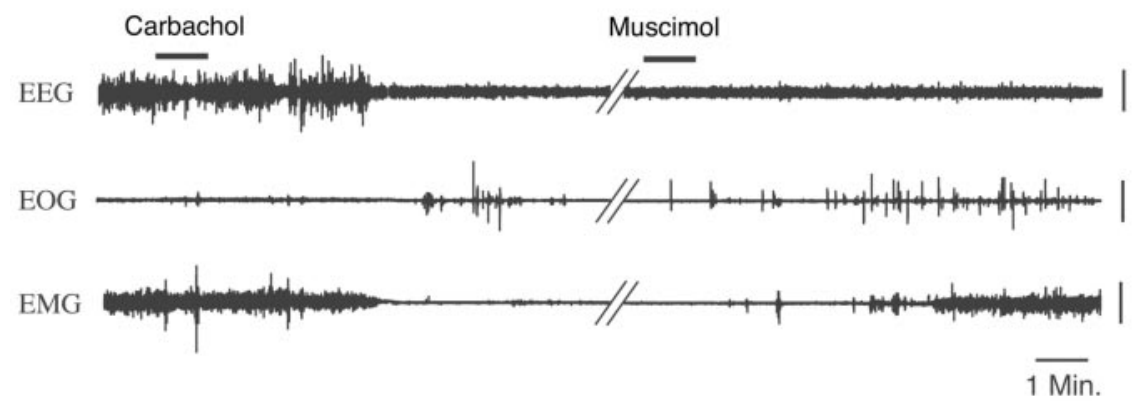

B. Carbachol and Muscimol

C. Carbachol and Saline

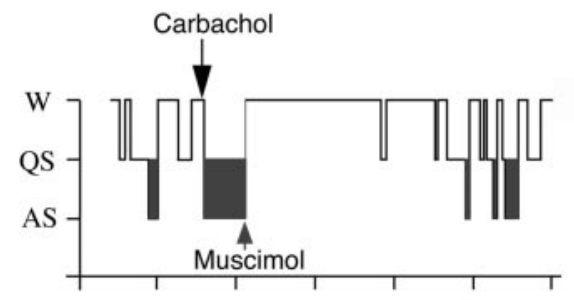

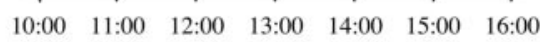

Time of Day

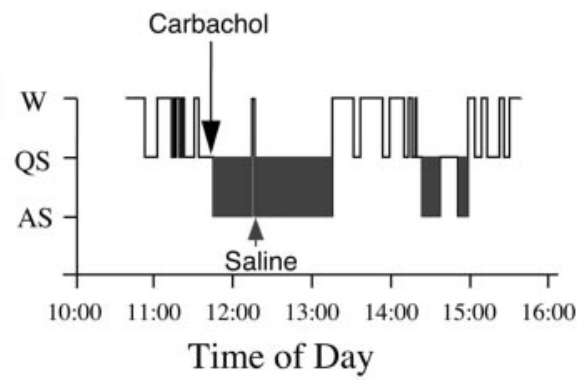

Figure 6. Carbachol-induced active sleep was suppressed by a subsequent injection of muscimol. $A$, Representative polygraphic recordings from an experimental session that consisted of the combined injection of muscimol and carbachol. An injection of carbachol into the NPO induced, with a short latency, a long-duration episode of active sleep. The injection of muscimol, which was performed $25 \mathrm{~min}$ after the injection of carbachol, immediately induced wakefulness. $B, C$, Hypnograms of recording sessions of the combined injection of carbachol and muscimol or carbachol and saline. The induction of active sleep by carbachol was suppressed by a subsequent injection of muscimol and a prolonged episode of wakefulness occurred after an injection of muscimol $(B)$. Control injections of saline into the same area of the NPO did not have an effect on carbachol-induced active sleep ( $C$ ). Vertical calibration bars, $100 \mu \mathrm{V}$.
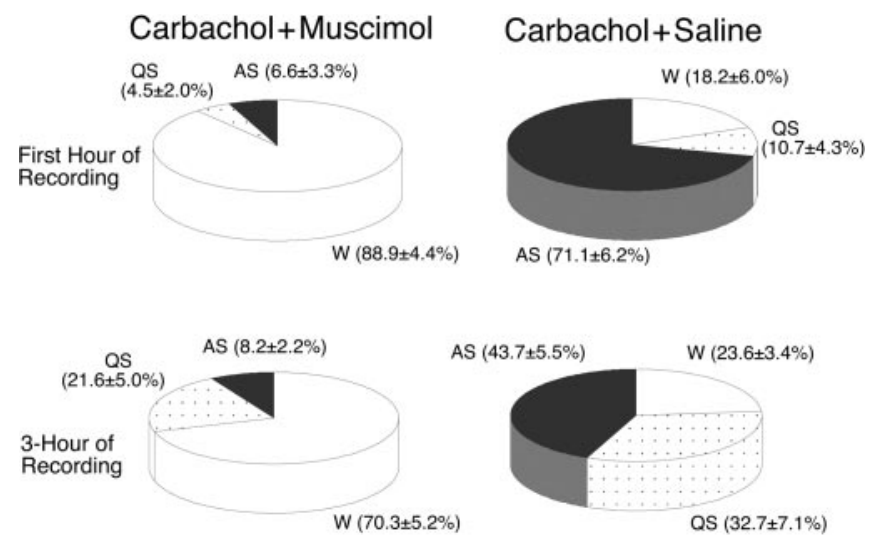

Figure 7. Pie charts presenting the mean percentage of time spent in wakefulness, quiet sleep, and active sleep during the first hour (top) and the $3 \mathrm{hr}$ recording period (bottom) after the combined injection of carbachol and muscimol or carbachol and saline, respectively. The injection of carbachol and muscimol significantly increased the percentage of time spent in wakefulness $(p<0.01)$ and significantly decreased the percentage of time spent in active sleep ( $p<0.01$ ) compared with these parameters after the injection of carbachol and saline during the first hour of recording. The injection of carbachol and muscimol also increased the percentage of time spent in wakefulness and decreased the percentage of time spent in active sleep ( $p<0.01$ ) during the $3 \mathrm{hr}$ recording period.

solution of muscimol followed by a carbachol injection $(23.7 \pm$ $4.0 \% ; n=4 ; p<0.05)$. The $10 \mathrm{~mm}$ concentration of muscimol produced the largest decrease in the percentage of time spent in active sleep.

\section{Effects of the injection of carbachol} followed by muscimol

The microinjection of carbachol into the NPO induced, with a short latency, active sleep and increased the time spent in this state, as reported previously (Baghdoyan et al., 1984, 1989, 1993; Yamamoto et al., 1990; Yamuy et al., 1993). However, the induction of active sleep by carbachol was suppressed immediately by a subsequent injection of muscimol; in addition, a prolonged episode of wakefulness occurred after the injection of muscimol. A control injection of saline did not effect active sleep, which was induced by a previous injection of carbachol (Fig. 6).

The effect of the combined injection of carbachol and muscimol on the percentage of time spent in sleep and waking states was examined during the first hour, as well as throughout the $3 \mathrm{hr}$ recording period (Fig. 7). During the first hour after the injection of carbachol and muscimol, there was a $388.5 \%$ increase in the percentage of time spent in wakefulness $(n=6)$, which was statistically significant compared with that after the injection of carbachol and saline $(n=5 ; p<0.01)$. This increase was accompanied by a $90.7 \%$ decrease in active sleep $(p<0.01)$.

There was also an increase of $197.9 \%$ $(p<0.01)$ in the time spent in wakefulness and a decrease of $81.2 \%(p<0.01)$ in active sleep during the $3 \mathrm{hr}$ recording period after the injection of carbachol and muscimol compared with that after the injection of carbachol and saline. The percentage change in the time spent in quiet sleep during the $3 \mathrm{hr}$ recording period was not statistically significant (Fig. 7).

The microinjection of muscimol induced wakefulness with a short latency (Fig. 8A). The mean latency of muscimol-induced wakefulness was $4.2 \pm 0.4 \mathrm{~min}$, which was significantly shorter than the mean latency of wakefulness that occurred after the injection of carbachol and saline $(44.6 \pm 5.0 \mathrm{~min} ; p<0.01)$.

Changes in the frequency of sleep and waking states that arose in conjunction with the combined injection of carbachol and muscimol into the NPO are presented in Figure $8 \mathrm{~B}$. After the injection of carbachol and muscimol, the frequency of active sleep was significantly reduced during the subsequent $3 \mathrm{hr}$ recording period (carbachol plus saline, $1.5 \pm 0.3$ episode/hr vs carbachol plus muscimol; $0.8 \pm 0.2$ episode/hr; $p<0.05)$. The frequency of wakefulness and quiet sleep was not significantly different.

The microinjection of carbachol followed by muscimol induced a long duration episode of wakefulness. The mean duration of the longest episode of wakefulness after the injection of carbachol and muscimol was $79.7 \pm 11.9$ min (Fig. 8C), which was significantly longer than the episodes of wakefulness that occurred after the injection of carbachol and saline (14.6 \pm 3.1 $\min ; p<0.01)$. On the other hand, the mean duration of the longest episode of active sleep after the injection of carbachol and muscimol was significantly reduced (carbachol plus muscimol, $9.4 \pm 2.1 \mathrm{~min}$ vs carbachol plus saline, $41.6 \pm 7.5 \mathrm{~min} ; p<0.01$ ). 


\section{A. Latency of Behavioral States}

1. Quiet Sleep

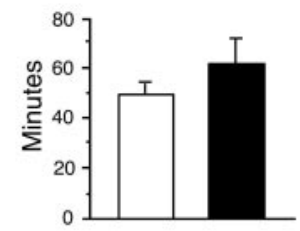

2. Wakefulness

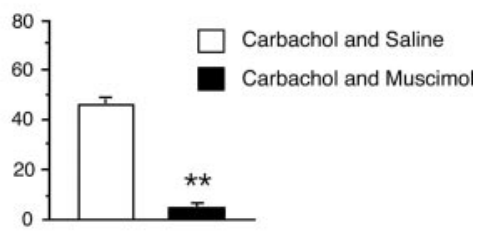

B. Frequency of Behavioral States

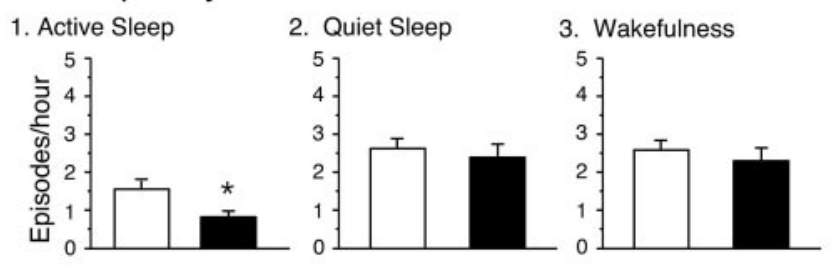

C. Duration of Behavioral States

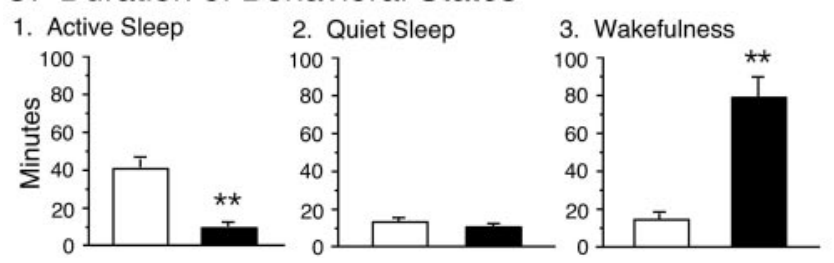

Figure 8. Effects of the combined injection of carbachol and muscimol on the latency, frequency, and duration of active sleep, quiet sleep, and wakefulness. Each bar represents the mean value; error bars indicate the SEM of each population. $A$, The effect of the injection of carbachol and muscimol on the latency of active sleep and wakefulness. Latency was measured from the second injection to the onset of the first episode of active sleep or wakefulness, respectively. The injection of carbachol and muscimol significantly increased the mean latency of active sleep and reduced the mean latency of wakefulness. $B$, The injection of carbachol and muscimol significantly reduced the frequency of active sleep. $C$, The injection of carbachol and muscimol significantly decreased the mean duration of active sleep and increased the mean duration of wakefulness. Asterisks indicate the levels of statistical significance of the difference between means; ${ }^{*} p<0.05 ;{ }^{* *} p<0.01$.

\section{Effects of the injection of scopolamine followed by bicuculline}

Representative polygraphic recordings and hypnograms that were obtained after the injection of scopolamine followed by bicuculline are presented in Figure 9. As we reported recently, the microinjection of bicuculline into the NPO results in the rapid induction of active sleep (Xi et al., 1999a,b, 2001). The induction of active sleep by bicuculline, however, was not blocked by a preinjection of scopolamine, which was delivered $30 \mathrm{~min}$ before the injection of bicuculline. However, in separate control experiments, the injection of scopolamine into the same area of the NPO successfully blocked the induction of active sleep by a following injection of carbachol, indicating that scopolamine antagonizes the cholinergic response.

The effect of the combined injection of scopolamine and bicuculline on the percentage of time spent in sleep and waking states was examined during the first hour, as well as throughout the $3 \mathrm{hr}$ recording period (Fig. 10). During the first hour after the injection of scopolamine and bicuculline $(n=6)$, there was a $750.6 \%$ increase in the percentage of time spent in active sleep compared with that after the injection of scopolamine and carbachol $(n=5$; $p<0.01$ ). This increase was accompanied by a significant decrease of 75.8 and $67.1 \%$ in the time spent in wakefulness and quiet sleep, respectively $(p<0.05)$. There was also a $193.2 \%$ increase in the time spent in active sleep during the $3 \mathrm{hr}$ recording period after the injection of scopolamine and bicuculline compared with that after the injection of scopolamine and carbachol $(p<0.01)$.

The microinjection of scopolamine followed by bicuculline induced active sleep with a short latency. The mean latency of bicuculline-induced active sleep was significantly shorter than that of wakefulness after the injection of scopolamine and carbachol (scopolamine plus bicuculline, $3.2 \pm 0.5 \mathrm{~min}, n=6$, vs scopolamine plus carbachol, $32.7 \pm 6.4 \mathrm{~min}, n=5 ; p<0.01$ ) (Fig. 11A).

The frequency of episodes of active sleep during the $3 \mathrm{hr}$ recording period after the injection of scopolamine and bicuculline was significantly greater than that after the injection of scopolamine and carbachol $[1.8 \pm 0.1$ episodes/hr (scopolamine plus bicuculline) vs $1.2 \pm 0.2$ episodes/hr (scopolamine plus carbachol); $p<0.05$ ] (Fig. $11 B$ ). On the other hand, the frequency of wakefulness was significantly reduced after the injection of scopolamine and bicuculline $[2.6 \pm 0.3$ episodes/hr (scopolamine plus bicuculline) vs $4.1 \pm 0.3$ episodes/hr (scopolamine plus carbachol); $p<0.05$ ] (Fig. 11B).

The microinjection of scopolamine followed by bicuculline resulted in long-duration episodes of active sleep. The mean duration of the longest episode of active sleep after the injection of scopolamine and bicuculline was significantly longer than that which occurred after the injection of scopolamine and carbachol $[31.1 \pm 5.1 \mathrm{~min}$ (scopolamine plus bicuculline) vs $8.8 \pm 2.0 \mathrm{~min}$ (scopolamine plus carbachol); $p<0.01$ ] (Fig. 11C).

\section{Discussion}

The present data demonstrate that, when muscimol, a $\mathrm{GABA}_{\mathrm{A}}$ receptor agonist, is injected into the NPO shortly before (20-30 min) the injection of carbachol into the same site, the active sleep-inducing effects of carbachol are blocked. The induction of active sleep by the microinjection of carbachol into the NPO is also suppressed by a subsequent injection of muscimol into the same area. The combined injection of muscimol and carbachol or carbachol and muscimol significantly increases the percentage of time spent in wakefulness and the duration of wakefulness, as well as the latency to active sleep; there is also a decrease in the percentage of time spent in active sleep, the frequency, and duration of active sleep compared with control injections of saline and carbachol or carbachol and saline. On the other hand, the injection of scopolamine into the NPO does not block the occurrence of active sleep that is induced by a subsequent injection of bicuculline into the same region. However, the injection of scopolamine effectively blocks the induction of active sleep by a subsequent injection of carbachol into the NPO. These results highlight the presence and importance of interactions between the pontine GABAergic system and the pontine cholinergic system in the generation and maintenance of active sleep and wakefulness.

There is no doubt that the pontine cholinergic system plays a key role in the generation of active sleep (for review, see Chase and Morales, 1990, 2000; Steriade and McCarley, 1990; Jones, 1991; Baghdoyan, 1997; Siegel, 2000). A number of studies, including ours, have shown that the administration of cholinergic agonists directly into the NPO readily evokes both individual elements of active sleep (e.g., atonia, ponto-geniculo-occipital waves, rapid eye movements, etc.), as well as the complete state of active sleep with all of its attendant physiological patterns of activity (George et al., 1964; Amatruda et al., 1975; Hobson et al., 
1983; Baghdoyan et al., 1984, 1989, 1993; Morales et al., 1987; Yamuy et al., 1993). Acetylcholine release in the NPO is significantly higher during active sleep than during quiet sleep or wakefulness (Kodama et al., 1990; Leonard and Lydic, 1997). In addition, the majority of the neurons in the NPO show an increased discharge rate during active sleep (McCarley and Hobson, 1971; Sakai, 1988; McCarley et al., 1995); many also increase their rate of discharge during waking movements (Siegel et al., 1977).

The NPO receives cholinergic innervation from the laterodorsal tegmental nucleus/pedunculopontine tegmental nucleus (LDT/PPT) (Mitani et al., 1988; Shiromani et al., 1988). Lesions of the cholinergic neurons in the LDT/PPT eliminate active sleep (Webster and Jones, 1988), whereas electrical stimulation of the LDT/PPT increases active sleep (Thakkar et al., 1996). Unit recordings studies in cats and rats demonstrate an increase in the discharge rate of a population of putatively cholinergic neurons in the LDT/PPT before and during active sleep (El Mansari et al., 1989; Steriade et al., 1990; Kayama et al., 1992). Together, these data strongly support the hypothesis that cells in the NPO function as executive neurons vis-à-vis the control of active sleep and that they, together with cholinergic neurons of the dorsolateral pons, play a critical role in the generation and maintenance of active sleep.

In experiments designed to further explore the neuronal basis for the control of active sleep and wakefulness, we began our examination of GABAergic effects within the pontine reticular formation with a question regarding the function(s) of the GABAergic innervation of cells in the NPO and their possible contribution to the modulation of sleep and wakefulness. In 1999, we reported that the microinjection of GABA and the $\mathrm{GABA}_{\mathrm{A}}$ agonist muscimol into the same area of the NPO of chronic cats, in which cholinergic agonists induce active sleep, results in prolonged episodes of wakefulness. Conversely, the injection of the corresponding $\mathrm{GABA}_{\mathrm{A}}$ antagonists, into the NPO, induces, with a short latency, long-duration episodes of active sleep (Xi et al., 1999a). Interestingly, the microinjection of glycine, another major inhibitory neurotransmitter in the CNS, and its antagonist strychnine into the same area of the NPO had no effect on the behavioral states of sleep and wakefulness (Xi et al., 1999b). These data demonstrate the unique importance of the pontine GABAergic system in the control of active sleep, as well as wakefulness.

The administration, into the NPO, of antisense against GAD, which blocks the synthesis of endogenous GABA, produces long periods $(>24 \mathrm{hr}$ ) of increased active sleep and decreased wakefulness (Xi et al., 1999b). In addition, microinjections of $\mathrm{GABA}_{\mathrm{B}}$ agonists and antagonists, into the NPO, yield effects that are similar but weaker than those responses induced by $\mathrm{GABA}_{\mathrm{A}}$ agonists and antagonists (Xi et al., 2001). We conclude that synaptic inhibition, mediated by $\mathrm{GABA}_{\mathrm{A}}$ receptors, plays a more important
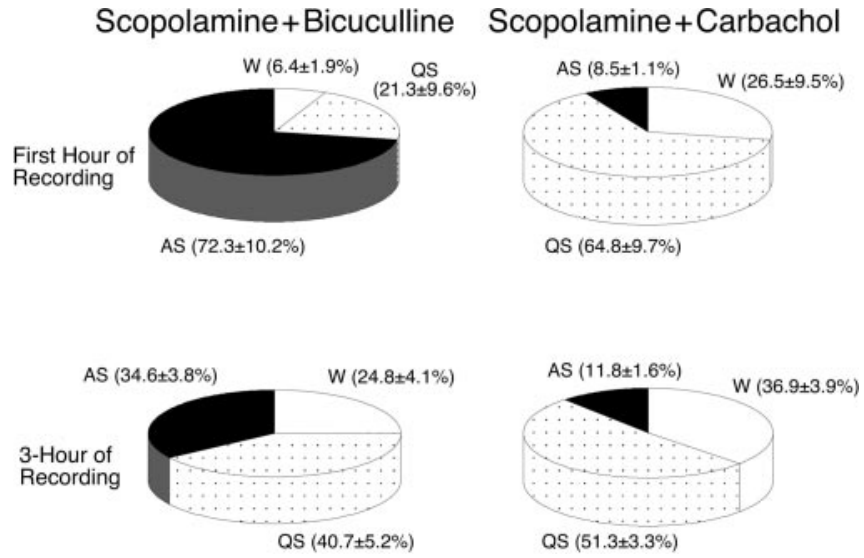

Figure 10. Pie charts presenting the mean percentage of time spent in wakefulness, quiet sleep, and active sleep during the first hour (top) and the $3 \mathrm{hr}$ recording period (bottom) after the combined injection of scopolamine and bicuculline or scopolamine and carbachol, respectively. Compared with the percentages observed after the injection of scopolamine and carbachol $(n=5)$, the injection of scopolamine did not block the increase in the percentage of time spent in active sleep $(p<0.01)$ or the reduction in the percentage of time spent in quiet sleep $(p<0.05)$ and wakefulness $(p<0.05)$ induced by bicuculline $(n=6)$ during the first hour of recording. During the $3 \mathrm{hr}$ recording period, the injection of scopolamine and bicuculline produced significant changes only in the percentage of the time spent in active sleep $(p<0.01)$.

role in the control of active sleep and wakefulness than the inhibitory synaptic processes that are mediated by $\mathrm{GABA}_{\mathrm{B}}$ receptors. $\mathrm{A}$ number of recent studies in rats and guinea pigs have also shown that the pontine administration of bicuculline enhances active 


\section{A. Latency of Behavioral States}

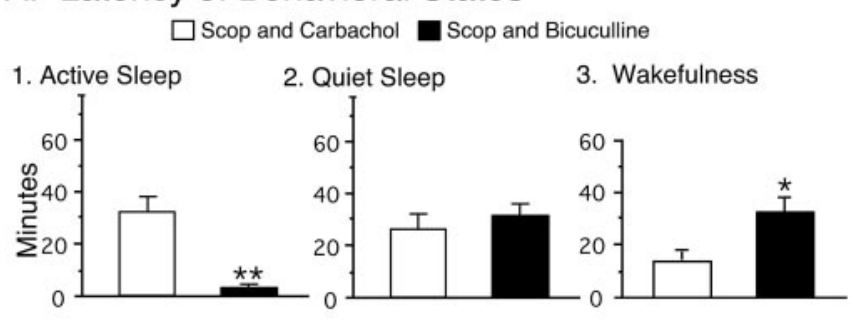

\section{B. Frequency of Behavioral States}

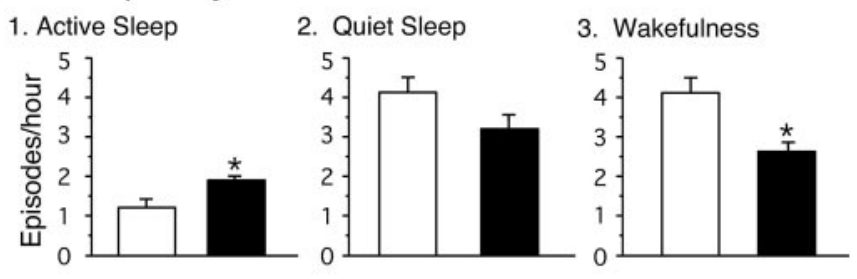

\section{Duration of Behavioral States}

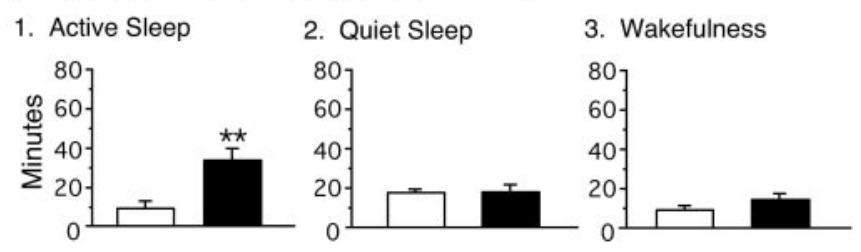

Figure 11. Effects of the injection of scopolamine and bicuculline on the latency, frequency, and duration of active sleep, quiet sleep, and wakefulness. Each bar represents the mean value; error bars indicate the SEM of each population. $A$, The injection of scopolamine and bicuculline significantly reduced the mean latency of active sleep $(p<0.01)$ and increased the mean latency of wakefulness $(p<0.05)$ compared with these parameters after the injection of scopolamine and carbachol. $B$, The injection of scopolamine and bicuculline significantly increased the frequency of active sleep $(p<0.05)$. C, The injection of scopolamine and bicuculline significantly increased the mean duration of active sleep. Asterisks indicate the levels of statistical significance of the difference between means; ${ }^{*} p<0.05$; ${ }^{* *} p<0.01$.

sleep and muscimol suppresses active sleep (Boissard et al., 2002; Torterolo et al., 2002; Pollock and Mistlberger, 2003; Sanford et al., 2003). Therefore, clearly a pontine GABAergic system functions to suppress active sleep and promote wakefulness.

We hypothesize that the pontine GABAergic system acts within the context of a "gating" mechanism in the NPO to control the generation and maintenance of active sleep and wakefulness. Accordingly, when GABAergic neurons are active, executive "active sleep-on" NPO neurons, which are responsible for the generation of active sleep, are inhibited. During wakefulness and quiet sleep, the activity of GABAergic processes in the NPO is both tonic and dominant compared with excitatory cholinergic drives; consequently, active sleep-on NPO neurons are tonically inhibited. Active sleep occurs when GABAergic synaptic transmission in the NPO is suppressed (i.e., not present); therefore, active sleep-on neurons are capable of being activated by cholinergic inputs.

The present data demonstrate that an injection of muscimol into the NPO is capable of blocking carbachol-induced active sleep and that the induction of active sleep by carbachol is suppressed by a subsequent injection of muscimol; however, an injection of scopolamine does not block a bicuculline-induced increase in active sleep. Therefore, we conclude that the above described gating mechanism exerts its effects postsynaptically by direct GABAergic inhibitory control of active sleep-on NPO neu- rons. On the other hand, a recent preliminary study has found that the microinfusion of bicuculline into the NPO produces an increase in the amount of ACh release in the cat (Baghdoyan et al., 2002). This increase was blocked by the coinfusion of muscimol. However, the infusion of muscimol alone had no effect on ACh release (Baghdoyan et al., 2002). These authors conclude that GABAergic transmission inhibits active sleep by inhibiting ACh release in the NPO, indicating that GABAergic gating mechanisms may also exert their effect presynaptically by controlling the release of ACh from cholinergic synaptic terminals.

The cell bodies of the pontine GABAergic system described in the present report may be located within the NPO (i.e., local interneurons) or outside of the NPO and project to this nucleus. Although these two alternatives are not mutually exclusive, our preliminary anatomical data support the concept that this system comprises GABAergic neurons that reside within the NPO (Xi et al., 1999c). Using c-fos immunocytochemistry as a functional marker of neuronal activity, we found a group of GABAergic neurons within the lateral part of the NPO that is activated during the waking state (Xi et al., 1999c). Consistent with these findings is a recent study that has shown that the number of activated GABAergic neurons (c-fos positive) decreases in the NPO of the rat during deprivation-induced REM sleep rebound (Maloney et al., 2000). However, a recent study by Boissard et al. (2003) suggests that GABAergic neurons, which are located in mesencephalic and rostral and caudal pontine reticular nuclei, project to the NPO; these neurons may also contribute to the pontine GABAergic system that is responsible for the regulation of active sleep and wakefulness.

Our demonstration of the dose-response effects of preinjections of different concentrations of muscimol in blocking the induction of active sleep by a subsequent injection of carbachol and the fact that the control injection of saline into the same area of the NPO did not block carbachol-induced active sleep clearly indicate that the effects of muscimol are pharmacologically specific. We, therefore, believe that the results described in the present study reflect interactions between cholinergic and GABAergic processes in the NPO. However, it is possible that there are also nonspecific effects that were produced by muscimol at high concentrations (Paredes and Agmo, 1992). Although we believe it unlikely, it has been suggested that some in vivo effects of muscimol might be attributable to the combined action of muscimol and its metabolites (Baraldi et al., 1979; Maggi and Enna, 1979).

In summary, the present results demonstrate the importance of interactions between cholinergic and GABAergic processes in the NPO with respect to the generation and maintenance of active sleep and wakefulness. In addition, these data also support the hypothesis that NPO neurons are involved in the generation of active sleep and that their excitatory cholinergic control is gated by a pontine GABAergic inhibitory system. The responsible gate mechanism is likely to exert its effects postsynaptically by direct GABAergic inhibition of NPO neurons. These findings provide the foundation for studies designed to identify the neuronal mechanisms that are responsible for the interactions among the neurotransmitter systems that control the behavioral states of sleep and wakefulness.

\section{References}

Amatruda III TT, Black DA, McKenna TM, McCarley RW, Hobson JA (1975) Sleep cycle control and cholinergic mechanisms: differential effects of carbachol injections at pontine brain stem sites. Brain Res 98:501-515. 
Baghdoyan HA (1997) Cholinergic mechanisms regulating REM sleep. In: Sleep sciences: integrating basic research and clinical practice. Monographs in clinical neuroscience (Schwartz WJ, ed), pp 88-116. Basel: Karger.

Baghdoyan HA, Rodrigo-Angulo ML, McCarley RW, Hobson JA (1984) Site-specific enhancement and suppression of desynchronized sleep signs following cholinergic stimulation of three brain-stem region. Brain Res 306:39-52.

Baghdoyan HA, Lydic R, Callaway CW, Hobson JA (1989) The carbacholinduced enhancement of desynchronized sleep signs is dose dependent and antagonized by centrally administered atropone. Neuropsychopharmacology 2:67-69.

Baghdoyan HA, Spotts JL, Snyder SG (1993) Simultaneous pontine and basal forebrain microinjections of carbachol suppress REM sleep. J Neurosci 13:229-242.

Baghdoyan HA, Vasquez J, Wilcox M, Wiesenauer K (2002) Pontine acetylcholine $(\mathrm{ACh})$ release is modulated by $\mathrm{GABA}_{\mathrm{A}}$ receptors. Soc Neurosci Abstr 28:870.3.

Baraldi M, Grandison L, Guidotti A (1979) Distribution and metabolism of muscimol in the brain and other tissues of the rat. Neuropharmacology 18:57-62.

Berman AL (1968) The brainstem of the cat. A cytoarchitectonic atlas with stereotaxic coordinates. Madison, WI: University of Wisconsin.

Boissard R, Gervasoni D, Schmidt MH, Barbagli B, Boissard R, Fort P, Gervasoni $\mathrm{D}$, Barbagli B, Luppi PH (2002) The rat ponto-medullary network responsible for paradoxical sleep onset and maintenance: a combined microinjection and functional neuroanatomical study. Euro J Neurosci 16:1959-1973.

Boissard R, Fort P, Gervasoni D, Barbagli B, Luppi PH (2003) Localization of the GABAergic and non-GABAergic neurons projecting to the sublaterodorsal nucleus and potentially gating paradoxical sleep onset. Euro J Neurosci 18:1627-1639.

Chase MH, Morales FR (1990) The atonia and myoclonia of active (REM) sleep. Annu Rev Psychol 41:557-584.

Chase MH, Morales FR (2000) Control of motoneurons during sleep. In: Principles and practice of sleep medicine (Kryger MH, Roth T, Dement WC, eds), pp 155-168. Philadelphia: Saunders.

El Mansari M, Sakai K, Jouvet M (1989) Unitary characteristics of presumptive tegmental neurons during the sleep-waking cycle in free moving cats. Exp Brain Res 76:519-529.

George R, Haslett WL, Jenden DJ (1964) A cholinergic mechanism in the brainstem reticular formation: induction of paradoxical sleep. Int J Neuropharmacol 3:541-552.

Hobson JA, Goldberg M, Vivaldi E, Riew D (1983) Enhancement of desynchronized sleep signs after pontine microinjection of the muscarinic agonist bethannechol. Brain Res 275:127-136.

Jones BE (1991) Paradoxical sleep and its chemical/structural substrates in the brain. Neuroscience 40:637-656.

Kayama Y, Ohta M, Jodo E (1992) Firing of "possibly" cholinergic neurons in the rat laterodorsal tegmental nucleus during sleep and wakefulness. Brain Res 569:210-220.

Kodama T, Takahashi Y, Honda Y (1990) Enhancement of acetylcholine release during paradoxical sleep in the dorsal tegmental field of the cat brain stem. Neurosci Lett 114:277-282.

Leonard TO, Lydic R (1997) Pontine nitric oxide modulates acetylcholine release, rapid eye movement sleep generation, and respiratory rate. J Neurosci 17:774-785.

Lydic R, Baghdoyan HA, Lorinc Z (1991) Microdialysis reveals enhanced pontine acetylcholine release during state-dependent respiratory depression. Am J Physiol 261:R766-R770.

Maggi A, Enna SJ (1979) Characteristics of muscimol accumulation in mouse brain after systemic administration. Neuropharmacology $18: 361-366$

Maloney KJ, Mainville L, Jones BE (2000) c-Fos expression in GABAergic, serotonergic, and other neurons of the pontomedullary reticular formation and raphe after paradoxical sleep deprivation and recovery. J Neurosci 20:4669-4679.

McCarley RW, Hobson JA (1971) Single neuron activity in cat gigantocellular tegmental field: selectively of discharge in desynchronized sleep. Science 174:1250-1252.
McCarley RW, Greene RW, Raining DG, Portas CM (1995) Brainstem neuromodulation and REM sleep. Semin Neurosci 7:341-354.

Mitani A, Ito K, Hallanger AH, Wainer BH, Kataoka K, McCarley RW (1988) Cholinergic projections from the laterodorsal and pedunculopontine tegmental nuclei to the pontine gigantocellular tegmental field in the cat. Brain Res 451:397-402.

Morales FR, Engelhardt JK, Soja PJ, Pereda AE, Chase MH (1987) Motoneuron properties during motor inhibition produced by microinjection of carbachol into the pontine reticular formation of the decerebrate cat. J Neurophysiol 57:1118-1129.

Paredes RG, Agmo A (1992) GABA and behavior: the role of receptor subtypes. Neurosci Biobehav Rev 16:145-170.

Pollock MS, Mistlberger RE (2003) Rapid eye movement sleep induction by microinjection of the GABA-A antagonist bicuculline into the dorsal subcoeruleus area of the rat. Brain Res 962:68-77.

Rye DB (1997) Contributions of the pedunculopontine region to normal and altered REM sleep. Sleep 20:757-788.

Sakai K (1988) Executive mechanisms of paradoxical sleep. Arch Ital Biol 126:239-257.

Sanford LD, Tang X, Xiao J, Ross RJ, Morrison AR (2003) GABAergic regulation of REM sleep in reticularis pontis oralis and caudalis in rats. J Neurophysiol 90:938-945.

Shiromani PJ, Armstrong DM, Gillin JC (1988) Cholinergic neurons from the dorsolateral pons project to the medial pons: a WGA-HRP and choline acetyltransferase immunohistochemical study. Neurosci Lett 95:19-23.

Siegel JM (2000) Brainstem mechanisms generating REM sleep. In: Principles and practice of sleep medicine (Kryger MH, Roth T, Dement WC, eds), pp 112-133. Philadelphia: Saunders.

Siegel JM, McGinty DJ, Breedlove SM (1977) Sleep and waking activity of pontine gigantocellular field neurons. Exp Neurol 56:553-573.

Steriade M, McCarley RW (1990) Brainstem control of wakefulness and sleep. New York: Plenum.

Steriade M, Datta S, Oakson G, Dossi RC (1990) Neuronal activities in brainstem cholinergic nuclei related to tonic activation processes in thalamocortical systems. J Neurosci 10:2541-2559.

Thakkar M, Portas C, McCarley RW (1996) Chronic low-amplitude electrical stimulation of the laterodorsal tegmental nucleus of freely moving cats increases REM sleep. Brain Res 723:223-227.

Torterolo P, Morales FR, Chase MH (2002) GABAergic processes controlling active sleep and wakefulness in the nucleus pontis oralis of the guinea pig. Sleep 25:171.

Ursin R, Sterman MB (1981) A manual for standardized scoring of sleep and waking states in the adult cats. Los Angeles: University of California, Los Angeles, Brain Information Service/Brain Research Institute.

Vanni-Mercier G, Sakai K, Lin JS, Jouvet M (1989) Mapping of cholinoceptive brainstem structures responsible for the generation of paradoxical sleep in the cat. Arch Ital Biol 127:133-164.

Velazquez-Moctezuma J, Shalauta M, Gillin JC, Shiromani P (1991) Cholinergic natagonists and REM sleep generation. Brain Res 543:175-179.

Webster HH, Jones BE (1988) Neurotoxic lesions of the dirsolateral pontomesencephalic tegmentum-cholinergic cell area in the cat. II. Effects upon sleep-waking states. Brain Res 458:285-302.

Xi MC, Morales FR, Chase MH (1999a) A GABAergic pontine reticular system is involved in the control of wakefulness and active sleep. Sleep Res Online 2:43-48.

Xi MC, Morales FR, Chase MH (1999b) Evidence that wakefulness and REM sleep are controlled by a GABAergic pontine mechanism. J Neurophysiol 82:2015-2019.

Xi MC, Morales FR, Chase MH (1999c) Activation of pontine GABAergic neurons during wakefulness. Soc Neurosci Abstr 25:460.6.

Xi MC, Morales FR, Chase MH (2001) Induction of wakefulness and inhibition of active (REM) sleep by GABAergic processes in the nucleus pontis oralis. Arch Ital Biol 139:125-145.

Yamamoto K, Mamelak AN, Quattrochi JJ, Hobson AH (1990) A cholinoceptive desynchronized sleep induction zone in the anterodorsal pontine tegmentum: locus of sensitive region. Neuroscience 39:270-293.

Yamuy J, Mancillias JR, Morales FR, Chase MH (1993) c-Fos expression in the pons and medulla of the cat during carbachol-induced active sleep. J Neurosci 13:2703-2718. 